\title{
Investigation of Benford's Law with YouTube Social Media Statistics
}

\author{
Chi-Wei Chen" ${ }^{1}$ Shao-Yu Yu and Hsin-Ye Chen\# \\ ${ }^{1}$ Taipei Fuhsing Private School, Taipei, Taiwan, R.O.C. \\ \#Advisor
}

$\underline{\text { ABSTRACT }}$

In this study, we used social media data to investigate Benford's Law. In our experimental analysis, we used three control variables: Total Subscriptions, Total Views, and Video Uploads of YouTube channels to verify if the data is artificial and whether or not it fits Benford's Law. We noticed how Total Subscriptions does not fit Benford's Law for the top 5000 most-subscribed channels, and Total Views doesn't fit for the top 5000 most-viewed channels. The reasons that cause this difference are further investigated in this paper. We also proposed a mathematical model to verify if other datasets fit Benford's Law. After curve fitting the experimental data, results revealed that closer a and $\mathrm{b}$ values in our mathematical model indicate that a dataset fits Benford's Law.

\section{Introduction}

The advent of modern technology and the Internet of Things has provided the public with more convenient methods for obtaining data and knowledge, thus increasing the importance of one's ability to discern true data from false data and rumors. During the past few decades, experts in the fields of Accounting and Statistics have utilized Benford's Law[1] to assess the reliability of data. Benford's Law is the result of physicist Frank Benford's observations across diverse sets of real life data in 1938, including the surface area of 335 rivers, the size of 3259 populations in the US, and the molecular mass of 1800 molecules, all of which show results that conform with the first-digit frequencies as described by Benford's Law: in a randomly generated and evenly distributed dataset, the frequencies of data having 1,2 , and 3 as their leading digit are $30.1 \%, 17.6 \%$, and $12.5 \%$, respectively, with the frequencies of all following numbers decreasing[2][3]. Today, Benford's Law is used to test the reliability of Presidential election voting counts, financial statements, and many other types of data[2][4]. Being an easily implemented test for evaluating data credibility, Benford's Law has been the topic of many research papers. However, the limits to the applicability of Benford's Law are much less than specific, resulting in many misinterpretations of data and misconceptions [4][5]. The consensus is that Benford's Law is applicable only to "randomly and uniformly distributed data". According to this rule of thumb, it makes sense for cheque numbers and ID numbers to not conform with Benford's Law, but this definition is often blurred when applied to other datasets, causing randomly generated data to not follow Benford's Law while others do. In addition, many researchers have proven Benford's Law under the assumption that the growth rate of data is proportionate to its current value, but not all datasets that follow Benford's Law have this property. In this research, we further discuss the applicability of Benford's Law to data from social media platforms and the mechanics behind Benford's Law.

\section{Review of Literature}

We wish to derive a mathematical model of Benford's Law through mathematical analysis. The following is the theoretical proof of Benford's Law: 
First, we hypothesize that there is a set of data, whose rate of growth is proportional to its value, which can be expressed with the following equation:

$$
\frac{\Delta N}{N \times \Delta t}=C
$$

We can analyze this equation to reach two conclusions:

The value of this data will grow exponentially, as presented by the following equation:

$$
N=N_{0} \times e^{c t}
$$

The time required for this data value to grow from $\mathrm{N} 1$ to $\mathrm{N} 2$ can be estimated by the following equation:

$$
t=c \times \log \frac{N_{2}}{N_{1}}
$$

This means that the time required for this data to grow from having 1 as its first-digit to having 2 as its first-digit is:

$$
t_{1}=c \times \log \frac{2}{1}
$$

According to the same equation, we can estimate the time needed for the first-digit to change from 2 to 3 :

$$
t_{2}=c \times \log \frac{3}{2}
$$

Following this pattern, if the value starts with $\mathrm{n}$ as its first-digit, the time needed for its first-digit to grow from $\mathrm{n}$ to $\mathrm{n}+1$ is:

$$
t_{n}=c \times \log \frac{(n+1)}{n}
$$

The time needed for this data to grow from 1 to 10 is:

$$
t=t_{1}+t_{2}+t_{3}+\cdots+t_{9}=c \times \log 10=c
$$

From the information above, we can deduce that the probability of a value's first-digit being 1 while it's growing from a single-digit number to a double-digit number or more can be calculated from the following equation:

$$
P_{1}=\frac{t_{1}}{t}=\log 2
$$

The probability of the first digit being 2 will follow this equation:

$$
P_{2}=\frac{t_{2}}{t_{1}}=\log \frac{3}{2}
$$

The probability of each number appearing at the first-digit is:

$$
\begin{gathered}
P_{1}=\log 2 P_{2}=\log \frac{3}{2} \\
P_{3}=\log \frac{4}{3} \\
\vdots \\
P_{9}=\log \frac{10}{9}
\end{gathered}
$$

The above is the theoretical proof of Benford's Law. 
From the proof above, we can know that the values of $P_{1}, P_{2}, P_{3}, P_{4}, P_{5}, P_{6}, P_{7}, P_{8}$, and $P_{9}$ are $0.301,0.176,0.125,0.097$, 0.079,0.067, 0.058, 0.051, and 0.046 and create Figure 1.

\begin{tabular}{|l|r|l|}
\hline$d$ & $P(d)$ & Relative size of $P(d)$ \\
\hline 1 & $30.1 \%$ & \\
\hline 2 & $17.6 \%$ & \\
\hline 3 & $12.5 \%$ & \\
\hline 4 & $9.7 \%$ & \\
\hline 5 & $7.9 \%$ & \\
\hline 6 & $6.7 \%$ & \\
\hline 7 & $5.8 \%$ & \\
\hline 8 & $5.1 \%$ & \\
\hline 9 & $4.6 \%$ & \\
\hline
\end{tabular}

Figure 1. The first digit distribution of Benford's Law

\section{Methods}

\section{Collect data of social media platforms}

In order to prove Benford's Law, we used YouTube statistics from the analytics website SocialBlade (https://socialblade.com) as an example. The three variables taken into consideration are Total Video Views, Video Uploads, and Total Subscriptions of top YouTube channels. With these three parameters as variables, we would discuss the applicability of Benford's Law by cross comparing the first-digit distribution of datasets with different parameters. First, we used Total Subscriptions as the control variable and found the top 5000 channels with the highest subscription numbers and their respective Video Uploads, Total Video Views, and Total Subscriptions, graphing the dataset's first digit distribution. Next, we gathered and graphed data with Video Uploads and Total Subscriptions as independent variables. Because of the online website's large dataset, we collected data with a Python Crawler program and recorded the data on Microsoft Excel sheets. The Crawler code is shown in Figure 2. 


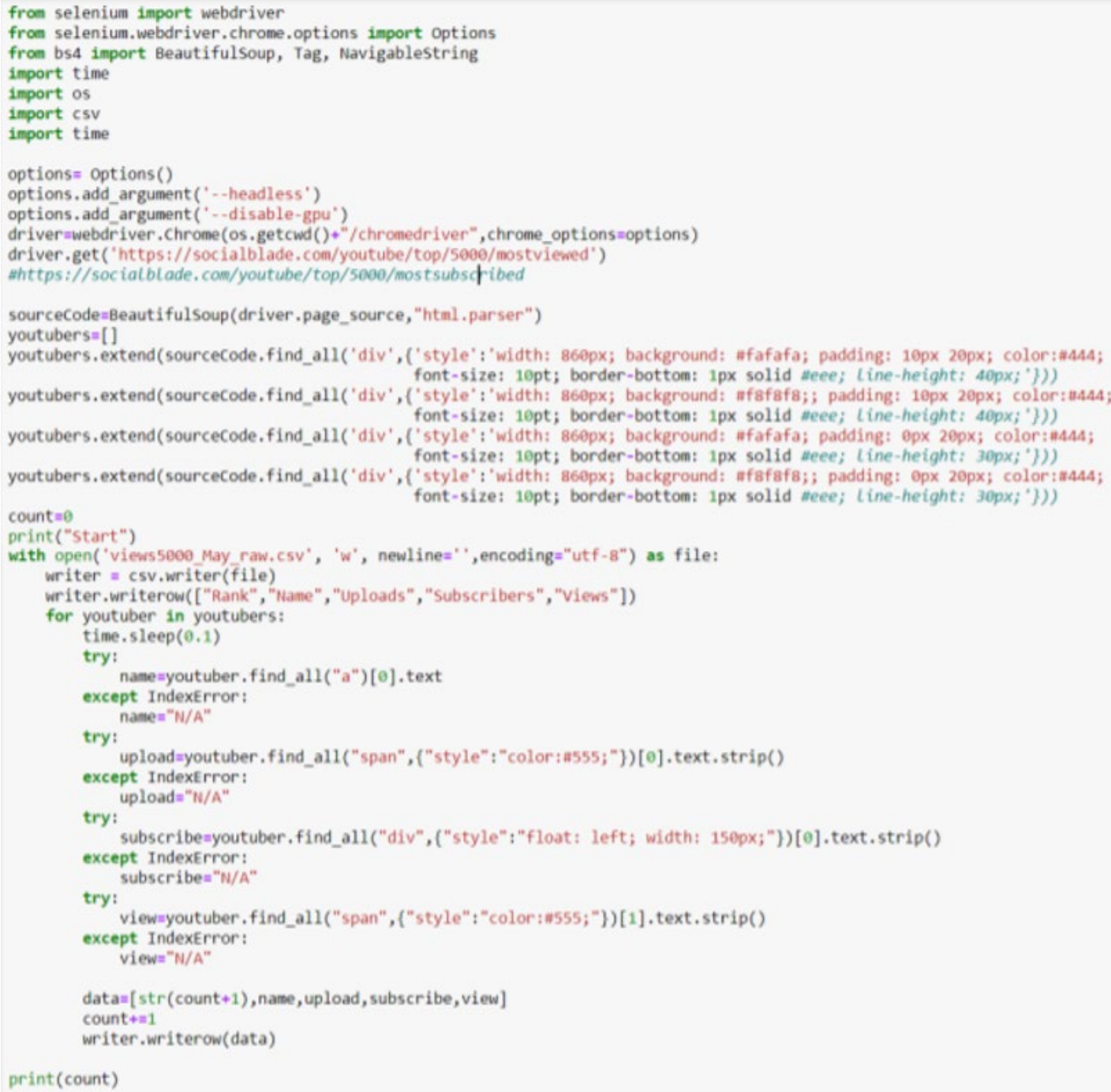

Figure 2. Python Crawler Code

\section{Mathematically fitting social media data}

In our research, we would like to develop a mathematical model to describe the trend of datasets that fit Benford's Law. We have chosen to fit the first-digit distribution data with $y=a e^{-b x}$ based on the hypothesis, as mentioned before, that the growth speed of a dataset's values is proportional to its current values.

By graphing the first-digit distribution of different groups and exponentially fitting them, we can find the a and $b$ values of the groups and compare them to Benford's Law. We hypothesize that groups with $a$ and $b$ values closer to calculated a and b values for Benford's Law will have a higher chance of fitting Benford's Law. After graphing the results and mathematically fitting them, we can analyze the errors of first-digit distribution data and a and $b$ values in order to evaluate whether or not a dataset follows Benford's Law, as a way of verifying whether or not a dataset is artificial. 


\section{Results}

With Total Subscriptions as the control variable

Firstly, we record the respective Video Uploads of the top 5000 Total Subscriptions channels and calculate the firstdigit distribution of these 5000 channels' Video Uploads. Figure 3a shows the first-digit distribution of Video Uploads for the top 5000 most-subscribed channels, showing distribution data for the top 1000 channels, top 2000 channels, top 3000 channels, top 4000 channels, top 5000 channels, and expected values according to Benford's Law. Figure 3b shows the raw data of distribution percentages, which shows that the first-distribution of the dataset has a decreasing trend that closely follows the expected values as calculated by Benford's Law.

(3a)

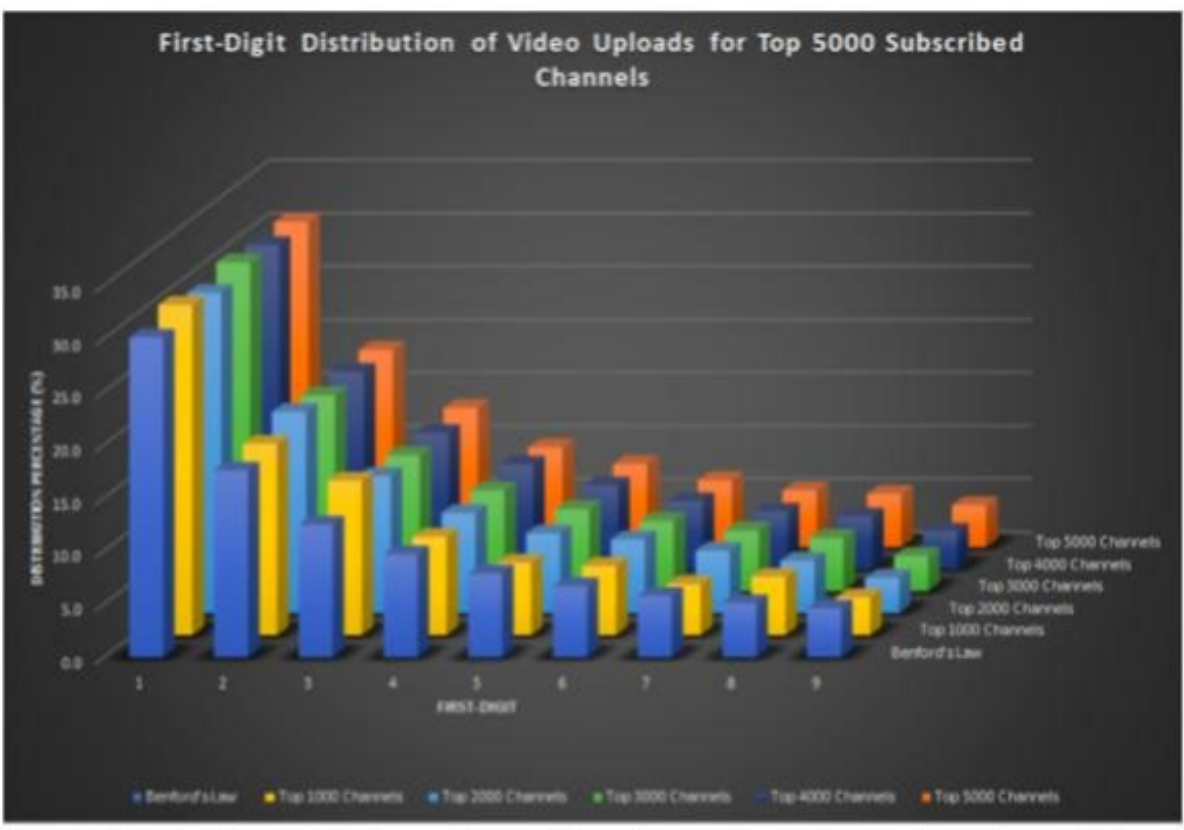

(3b)

\begin{tabular}{|l|l|l|l|l|l|l|l|l|l|}
\hline First-Digit Distribution (\%) & 1 & 2 & 3 & 4 & 5 & 6 & 7 & 8 & 9 \\
\hline Benford's Law Distribution & 30.1 & 17.6 & 12.5 & 9.7 & 7.9 & 6.7 & 5.8 & 5.1 & 4.6 \\
\hline Top 1000 Distribution & 31.1 & 18.0 & 14.5 & 9.2 & 6.8 & 6.5 & 4.8 & 5.5 & 3.6 \\
\hline Top 2000 Distribution & 30.2 & 19.0 & 12.9 & 9.4 & 7.5 & 6.9 & 5.9 & 4.9 & 3.3 \\
\hline Top 3000 Distribution & 30.9 & 18.4 & 12.8 & 9.5 & 7.7 & 6.6 & 5.7 & 5.0 & 3.3 \\
\hline Top 4000 Distribution & 30.5 & 18.6 & 12.8 & 9.9 & 7.9 & 6.4 & 5.4 & 5.0 & 3.5 \\
\hline Top 5000 (All data) & 30.6 & 18.6 & 13.1 & 9.4 & 7.8 & 6.3 & 5.3 & 5.0 & 3.9 \\
\hline
\end{tabular}

Figure 3 (a) First-digit distribution of video uploads compared to Benford's Law distribution for top 1000, 2000, 3000,4000 , and 5000 subscribed channels (upper) and (b) the experimental result corresponding to upper graph (lower). 
Secondly, we record the respective Total Video Views of the top 5000 Total Subscriptions channels and calculate the first-digit distribution of these 5000 channels' Total Video Views. Figure 4a shows the first-digit distribution of Total Video Views for the top 5000 most-subscribed channels, showing distribution data for the top 1000 channels, top 2000 channels, top 3000 channels, top 4000 channels, top 5000 channels, and expected values according to Benford's Law. Figure 4b shows the raw data of distribution percentages, which shows that the first-distribution of the dataset has a decreasing trend that closely follows the expected values as calculated by Benford's Law.

(4a)

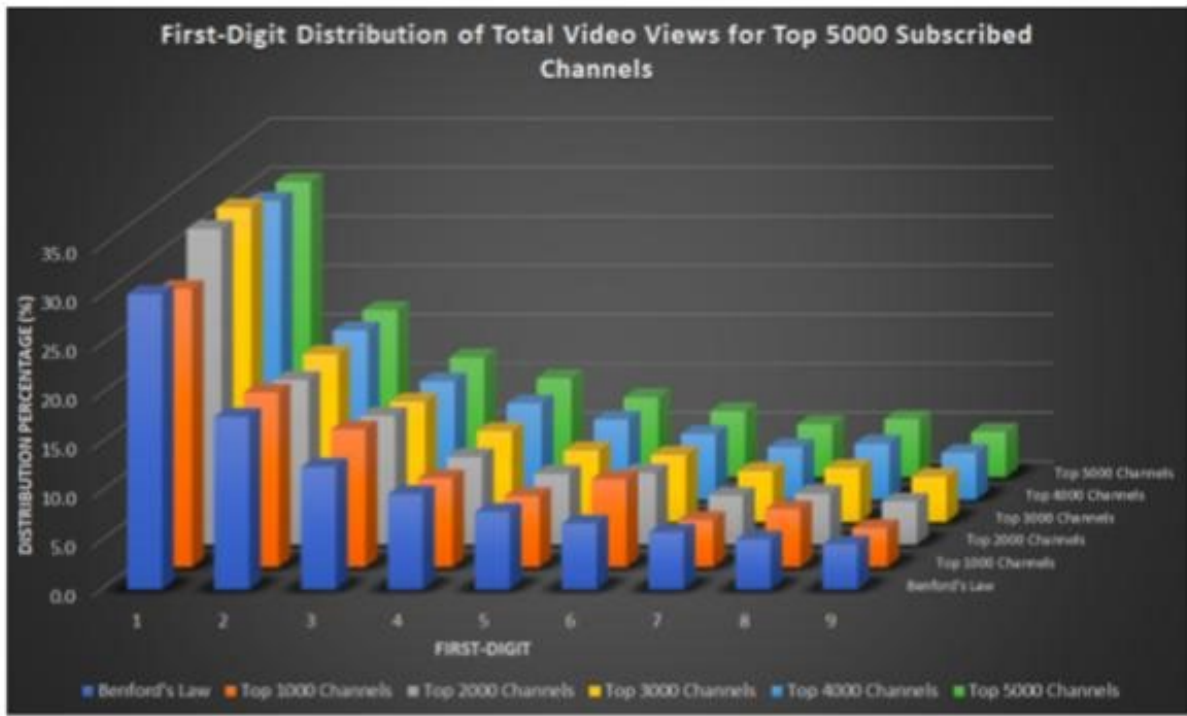

(4b)

\begin{tabular}{|l|l|l|l|l|l|l|l|l|l|}
\hline First-Digit Distribution (\%) & 1 & 2 & 3 & 4 & 5 & 6 & 7 & 8 & 9 \\
\hline Benford's Law Distribution & 30.1 & 17.6 & 12.5 & 9.7 & 7.9 & 6.7 & 5.8 & 5.1 & 4.6 \\
\hline Top 1000 Distribution & 28.4 & 17.8 & 14.0 & 9.0 & 7.2 & 8.9 & 4.8 & 5.9 & 4.0 \\
\hline Top 2000 Distribution & 32.2 & 16.8 & 13.1 & 8.9 & 7.2 & 7.3 & 5.0 & 5.2 & 4.4 \\
\hline Top 3000 Distribution & 32.1 & 17.1 & 12.3 & 9.2 & 7.3 & 6.8 & 5.2 & 5.5 & 4.6 \\
\hline Top 4000 Distribution & 30.5 & 17.2 & 12.0 & 9.8 & 8.1 & 6.7 & 5.3 & 5.7 & 4.7 \\
\hline Top 5000 (All data) & 30.1 & 17.0 & 12.2 & 10.1 & 8.1 & 6.7 & 5.4 & 5.9 & 4.6 \\
\hline
\end{tabular}

Figure 4 (a) First-digit distribution of total video views compared to Benford's Law distribution for top 1000, 2000, 3000, 4000, and 5000 subscribed channels (upper) and (b) the experimental result corresponding to upper graph (lower).

Thirdly, we record the respective Total Subscriptions of the top 5000 Total Subscriptions channels and calculate the first-digit distribution of these 5000 channels' Total Subscriptions. Figure 5a shows the first-digit distribution of Total Subscriptions for the top 5000 most-subscribed channels, showing distribution data for the top 1000 channels, top 2000 channels, top 3000 channels, top 4000 channels, top 5000 channels, and expected values according to Benford's Law. Figure $\mathbf{5 b}$ shows the raw data of distribution percentages, which shows that the first-distribution of the dataset does not follow Benford's Law. For example, the highest distribution percentage of the top 1000 channels group did occur at 1, but it didn't fully follow the decreasing trend of Benford's Law. The distribution percentage decreased from first-digit 1 to first-digit 6 , but the distribution percentage increased back to $16.5 \%$ at first-digit 7 . The other groups had similar deviations from Benford's Law, sometimes with the highest distribution percentage not occurring at first-digit 1 or with both increasing and decreasing trends while the first-digit number increased. 
(5a)

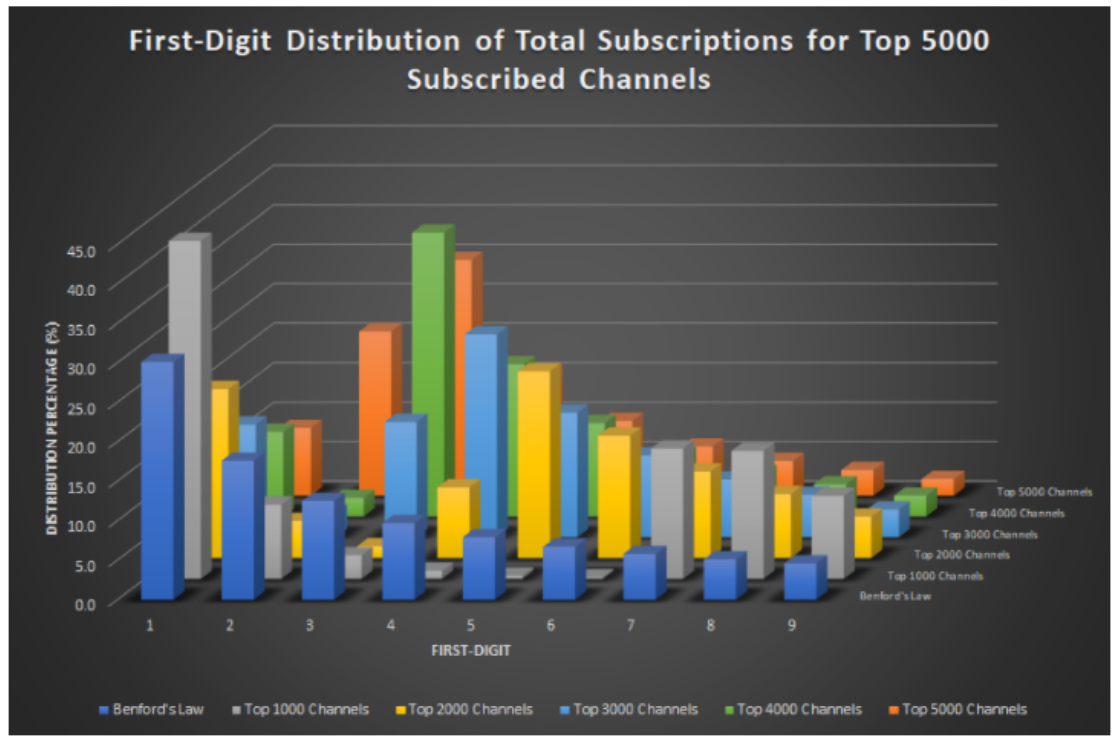

(5b)

\begin{tabular}{|l|l|l|l|l|l|l|l|l|l|}
\hline First-Digit Distribution (\%) & 1 & 2 & 3 & 4 & 5 & 6 & 7 & 8 & 9 \\
\hline Benford's Law Distribution & 30.1 & 17.6 & 12.5 & 9.7 & 7.9 & 6.7 & 5.8 & 5.1 & 4.6 \\
\hline Top 1000 Distribution & 42.8 & 9.4 & 3.0 & 1.0 & 0.4 & 0.2 & 16.5 & 16.2 & 10.5 \\
\hline Top 2000 Distribution & 21.4 & 4.7 & 1.5 & 9.0 & 23.7 & 15.5 & 11.0 & 8.1 & 5.3 \\
\hline Top 3000 Distribution & 14.3 & 3.1 & 14.6 & 25.7 & 15.8 & 10.3 & 7.3 & 5.4 & 3.5 \\
\hline Top 4000 Distribution & 10.7 & 2.4 & 36.0 & 19.3 & 11.8 & 7.8 & 5.5 & 4.1 & 2.6 \\
\hline Top 5000 (All data) & 8.6 & 20.8 & 29.8 & 15.4 & 9.5 & 6.2 & 4.4 & 3.2 & 2.1 \\
\hline
\end{tabular}

Figure 5 (a) First-digit distribution of total subscriptions compared to Benford's Law distribution for top 1000, 2000, 3000, 4000, and 5000 subscribed channels (upper) and (b) the experimental result corresponding to upper graph (lower).

\section{With Total Views as the control variable}

Firstly, we record the respective Video Uploads of the top 5000 Total Views channels and calculate the first-digit distribution of these 5000 channels' Video Uploads. Figure 6a shows the first-digit distribution of Video Uploads for the top 5000 most-viewed channels, showing distribution data for the top 1000 channels, top 2000 channels, top 3000 channels, top 4000 channels, top 5000 channels, and expected values according to Benford's Law. Figure $6 \mathbf{b}$ shows the raw data of distribution percentages, which shows that the first-distribution of the dataset has a decreasing trend that closely follows the expected values as calculated by Benford's Law. 
(6a)

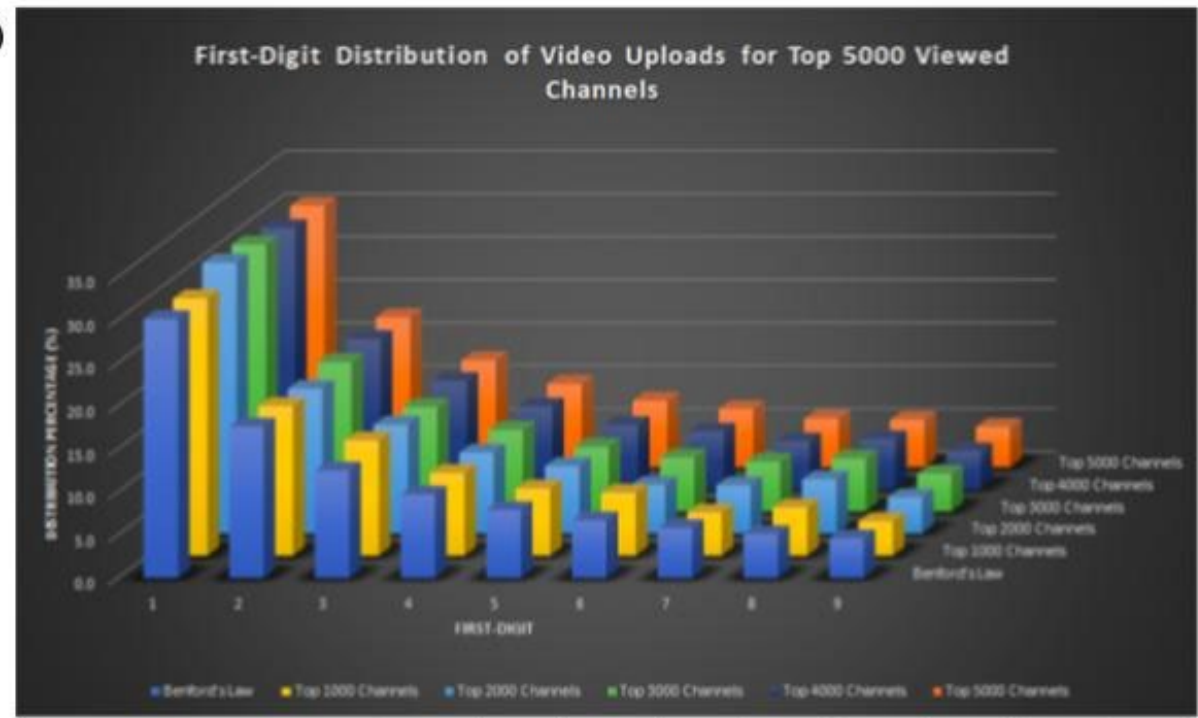

(6b)

\begin{tabular}{|l|l|l|l|l|l|l|l|l|l|}
\hline First-Digit Distribution (\%) & 1 & 2 & 3 & 4 & 5 & 6 & 7 & 8 & 9 \\
\hline Benford's Law Distribution & 30.1 & 17.6 & 12.5 & 9.7 & 7.9 & 6.7 & 5.8 & 5.1 & 4.6 \\
\hline Top 1000 Distribution & 29.9 & 17.4 & 13.4 & 9.6 & 7.8 & 7.3 & 5.0 & 5.6 & 4.0 \\
\hline Top 2000 Distribution & 31.5 & 16.9 & 12.7 & 9.4 & 7.9 & 5.7 & 5.6 & 6.3 & 4.2 \\
\hline Top 3000 Distribution & 31.0 & 17.3 & 12.1 & 9.5 & 7.5 & 6.3 & 5.8 & 6.2 & 4.4 \\
\hline Top 4000 Distribution & 30.4 & 17.5 & 12.6 & 9.6 & 7.5 & 6.7 & 5.6 & 5.8 & 4.4 \\
\hline Top 5000 (All data) & 30.4 & 17.4 & 12.4 & 9.6 & 7.7 & 6.7 & 5.6 & 5.5 & 4.6 \\
\hline
\end{tabular}

Figure 6 (a) First-digit distribution of video uploads compared to Benford's Law distribution for top 1000, 2000, 3000,4000 , and 5000 subscribed channels (upper) and (b) the experimental result corresponding to upper graph (lower).

Secondly, we record the respective Total Views of the top 5000 Total Views channels and calculate the first-digit distribution of these 5000 channels' Total Views. Figure 7a shows the first-digit distribution of Total Video Views for the top 5000 most-viewed channels, showing distribution data for the top 1000 channels, top 2000 channels, top 3000 channels, top 4000 channels, top 5000 channels, and expected values according to Benford's Law. Figure 7b shows the raw data of distribution percentages, which shows that the first-distribution of the dataset does not follow Benford's Law. For example, the highest distribution percentage of the top 1000 channels group occurred at first-digit 3 instead of 1, and it had an increasing trend from 1 to 3 and a decreasing trend from 3 to 9 . The other groups had similar deviations from Benford's Law, sometimes with the highest distribution percentage not occurring at first-digit 1 or with both increasing and decreasing trends while the first-digit number increased. 
(7a)

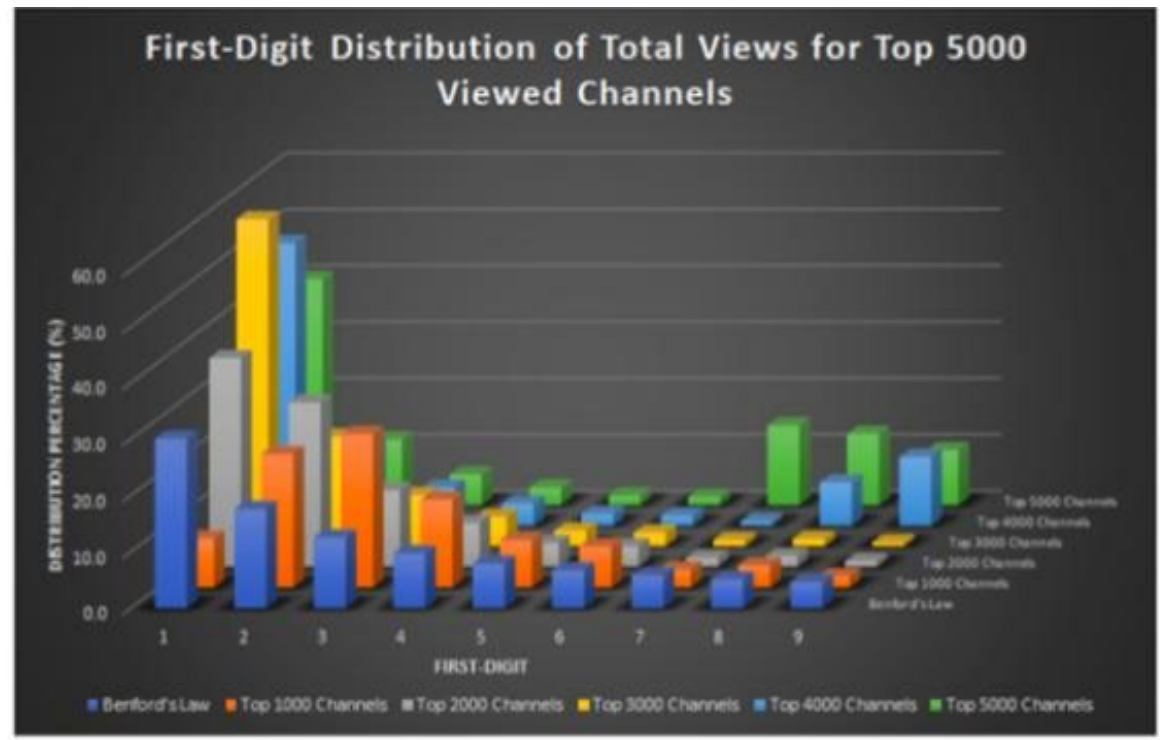

(7b)

\begin{tabular}{|l|l|l|l|l|l|l|l|l|l|}
\hline First-Digit Distribution (\%) & 1 & 2 & 3 & 4 & 5 & 6 & 7 & 8 & 9 \\
\hline Benford's Law Distribution & 30.1 & 17.6 & 12.5 & 9.7 & 7.9 & 6.7 & 5.8 & 5.1 & 4.6 \\
\hline Top 1000 Distribution & 29.5 & 13.4 & 11.1 & 8.9 & 9.6 & 7.6 & 8.6 & 6.6 & 4.8 \\
\hline Top 2000 Distribution & 24.7 & 15.8 & 14.4 & 11.5 & 9.9 & 8.3 & 6.3 & 5.4 & 3.7 \\
\hline Top 3000 Distribution & 25.4 & 17.7 & 15.3 & 11.3 & 9.3 & 7.3 & 5.5 & 4.8 & 3.5 \\
\hline Top 4000 Distribution & 26.7 & 19.3 & 15.6 & 10.2 & 8.3 & 6.7 & 5.3 & 4.4 & 3.4 \\
\hline Top 5000 (All data) & 40.1 & 11.7 & 5.4 & 3.1 & 1.7 & 1.5 & 14.1 & 12.6 & 9.7 \\
\hline
\end{tabular}

Figure 7 (a) First-digit distribution of total views compared to Benford's Law distribution for top 1000, 2000, 3000, 4000, and 5000 viewed channels (upper) and (b) the experimental result corresponding to upper graph (lower)

Thirdly, we record the respective Total Subscriptions of the top 5000 Total Views channels and calculate the firstdigit distribution of these 5000 channels' Total Subscriptions. Figure 8a shows the first-digit distribution of Total Subscriptions for the top 5000 most-viewed channels, showing distribution data for the top 1000 channels, top 2000 channels, top 3000 channels, top 4000 channels, top 5000 channels, and expected values according to Benford's Law. Figure $\mathbf{8 b}$ shows the raw data of distribution percentages, which shows that the first-distribution of the dataset has a decreasing trend that closely follows the expected values as calculated by Benford's Law. 
(8a)

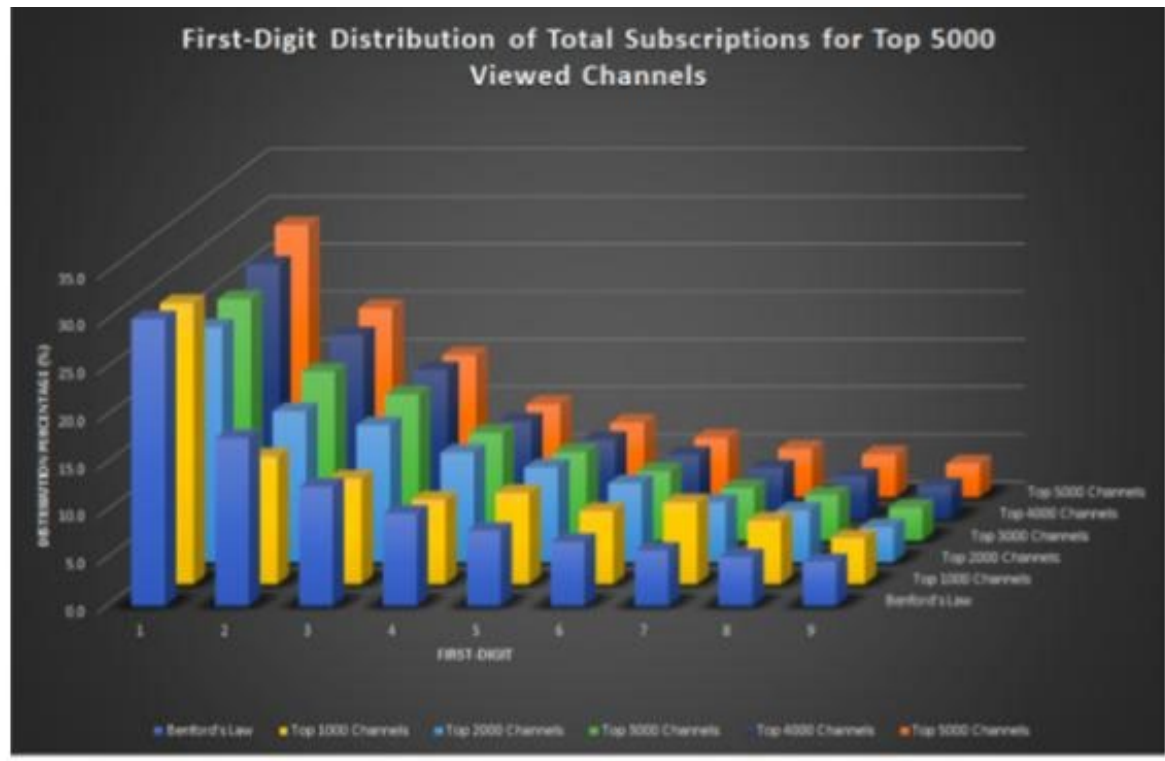

(8b)

\begin{tabular}{|l|l|l|l|l|l|l|l|l|l|}
\hline First-Digit Distribution (\%) & 1 & 2 & 3 & 4 & 5 & 6 & 7 & 8 & 9 \\
\hline Benford's Law Distribution & 30.1 & 17.6 & 12.5 & 9.7 & 7.9 & 6.7 & 5.8 & 5.1 & 4.6 \\
\hline Top 1000 Distribution & 29.5 & 13.4 & 11.1 & 8.9 & 9.6 & 7.6 & 8.6 & 6.6 & 4.8 \\
\hline Top 2000 Distribution & 24.7 & 15.8 & 14.4 & 11.5 & 9.9 & 8.3 & 6.3 & 5.4 & 3.7 \\
\hline Top 3000 Distribution & 25.4 & 17.7 & 15.3 & 11.3 & 9.3 & 7.3 & 5.5 & 4.8 & 3.5 \\
\hline Top 4000 Distribution & 26.7 & 19.3 & 15.6 & 10.2 & 8.3 & 6.7 & 5.3 & 4.4 & 3.4 \\
\hline Top 5000 (All data) & 28.5 & 19.8 & 14.9 & 9.7 & 7.8 & 6.2 & 5.0 & 4.5 & 3.5 \\
\hline
\end{tabular}

Figure 8 (a) First-digit distribution of total subscriptions compared to Benford's Law distribution for top 1000, 2000, 3000, 4000, and 5000 viewed channels (upper) and (b) the experimental result corresponding to upper graph (lower)

With Video Uploads as the control variable

Because SocialBlade doesn't provide rankings for the top 5000 channels with the highest Video Uploads, we are unable to conduct analysis with Video Uploads as the control variable. Nevertheless, we have observed the pattern that parameter A will not conform with Benford's Law while parameter B and C will, where A is used as the groups' control variable. Therefore, we can hypothesize that Total Subscriptions and Total Views will conform with Benford's Law while Video Uploads will not conform with Benford's Law, where Video Uploads is the control variable.

\section{Discussion}

Error of First-Digit Distribution in Comparison with Expected values

\section{Most-Subscribed Channels}


channels had a minimum of $0.189 \%$ error and a maximum of $28.381 \%$ error, with the average error being $6.237 \%$. The error of the first-digit distribution of the top 3000 most-subscribed YouTube channels had a minimum of $1.270 \%$ error and a maximum of $28.099 \%$ error, with the average error being $5.260 \%$. The error of the first-digit distribution of the top 4000 most-subscribed YouTube channels had a minimum of $0.261 \%$ error and a maximum of $22.891 \%$ error, with the average error being 5.362\%. The error of the first-digit distribution of the top 5000 most-subscribed YouTube channels had a minimum of $1.268 \%$ error and a maximum of $14.559 \%$ error, with the average error being $5.226 \%$. These results are also shown in Figure 9.

\begin{tabular}{|c|c|c|c|}
\hline $\begin{array}{c}\text { Most-Subscribed } \\
\text { Channels }\end{array}$ & Minimum Error (\%) & Maximum Error (\%) & Average Error (\%) \\
\hline Top 1000 & 2.203 & 22.676 & 10.122 \\
\hline Top 2000 & 0.189 & 28.381 & 6.237 \\
\hline Top 3000 & 1.270 & 28.099 & 5.260 \\
\hline Top 4000 & 0.261 & 22.891 & 5.362 \\
\hline Top 5000 & $\mathbf{1 . 2 6 8}$ & $\mathbf{1 4 . 5 5 9}$ & $\mathbf{5 . 2 2 6}$ \\
\hline
\end{tabular}

Figure 9. Error of first-digit distributions of Video Uploads for top 5000 most-subscribed channels

Next, we evaluated the error of observed Total Views first-digit distribution for the top 1000 most-subscribed YouTube channels. From the digits 1 to 9, the error of each digit distribution was between 1.049\% and 33.479\%, with the average error being $12.779 \%$. The error of the first-digit distribution of the top 2000 most-subscribed YouTube channels had a minimum of $1.368 \%$ error and a maximum of $14.361 \%$ error, with the average error being $7.051 \%$. The error of the first-digit distribution of the top 3000 most-subscribed YouTube channels had a minimum of $0.219 \%$ error and a maximum of $10.971 \%$ error, with the average error being $4.869 \%$. The error of the first-digit distribution of the top 4000 most-subscribed YouTube channels had a minimum of $0.548 \%$ error and a maximum of $11.278 \%$ error, with the average error being 3.811\%. The error of the first-digit distribution of the top 5000 most-subscribed YouTube channels had a minimum of $0.049 \%$ error and a maximum of $15.597 \%$ error, with the average error being 3.991\%. These results are also shown in Figure 10.

\begin{tabular}{|c|c|c|c|}
\hline $\begin{array}{c}\text { Most-Subscribed } \\
\text { Channels }\end{array}$ & Minimum Error (\%) & Maximum Error (\%) & Average Error (\%) \\
\hline Top 1000 & 1.049 & 33.479 & 12.779 \\
\hline Top 2000 & 1.368 & 14.361 & 7.051 \\
\hline Top 3000 & 0.219 & 10.971 & 4.869 \\
\hline Top 4000 & 0.548 & 11.278 & 3.811 \\
\hline Top 5000 & $\mathbf{0 . 0 4 9}$ & $\mathbf{1 5 . 5 9 7}$ & $\mathbf{3 . 9 9 1}$ \\
\hline
\end{tabular}

Figure 10. Error of first-digit distributions of Total Views for top 5000 most-subscribed channels

Thirdly, we evaluated the error of observed Total Subscriptions first-digit distribution for the top 1000 most-subscribed YouTube channels. From the digits 1 to 9 , the error of each digit distribution was between $42.193 \%$ and 
$217.167 \%$, with the average error being $108.535 \%$. The error of the first-digit distribution of the top 2000 mostsubscribed YouTube channels had a minimum of $7.732 \%$ error and a maximum of $199.367 \%$ error, with the average error being $76.710 \%$. The error of the first-digit distribution of the top 3000 most-subscribed YouTube channels had a minimum of $5.882 \%$ error and a maximum of $164.948 \%$ error, with the average error being $58.446 \%$. The error of the first-digit distribution of the top 4000 most-subscribed YouTube channels had a minimum of 5.603\% error and a maximum of $187.600 \%$ error, with the average error being $5.362 \%$. The error of the first-digit distribution of the top 5000 most-subscribed YouTube channels had a minimum of 7.463\% error and a maximum of $138.720 \%$ error, with the average error being 47.771\%. These results are also shown in Figure 11.

\begin{tabular}{|c|c|c|c|}
\hline $\begin{array}{c}\text { Most-Subscribed } \\
\text { Channels }\end{array}$ & Minimum Error (\%) & Maximum Error (\%) & Average Error (\%) \\
\hline Top 1000 & 42.193 & 217.167 & 108.535 \\
\hline Top 2000 & 7.732 & 199.367 & 76.710 \\
\hline Top 3000 & 5.882 & 164.948 & 58.446 \\
\hline Top 4000 & 5.603 & 187.600 & 63.544 \\
\hline Top 5000 & $\mathbf{7 . 4 6 3}$ & $\mathbf{1 3 8 . 7 2 0}$ & $\mathbf{4 7 . 7 7 1}$ \\
\hline
\end{tabular}

Figure 11. Error of first-digit distributions of Total Subscriptions for top 5000 most-subscribed channels

\section{Most-Viewed Channels}

First, we evaluated the error of observed Video Uploads first-digit distribution for the top 1000 most-viewed YouTube channels. From the digits 1 to 9 , the error of each digit distribution was between $0.664 \%$ and $13.793 \%$, with the average error being $6.321 \%$. The error of the first-digit distribution of the top 2000 most-viewed YouTube channels had a minimum of $0.633 \%$ error and a maximum of $23.529 \%$ error, with the average error being $7.423 \%$. The error of the first-digit distribution of the top 3000 most-viewed YouTube channels had a minimum of $0.575 \%$ error and a maximum of $20.915 \%$ error, with the average error being $5.326 \%$. The error of the first-digit distribution of the top 4000 most-viewed YouTube channels had a minimum of $0.001 \%$ error and a maximum of $14.216 \%$ error, with the average error being 3.530\%. The error of the first-digit distribution of the top 5000 most-viewed YouTube channels had a minimum of $0.597 \%$ error and a maximum of $7.451 \%$ error, with the average error being $2.004 \%$. These results are also shown in Figure 12.

\begin{tabular}{|c|c|c|c|}
\hline $\begin{array}{c}\text { Most-Subscribed } \\
\text { Channels }\end{array}$ & Minimum Error (\%) & Maximum Error (\%) & Average Error (\%) \\
\hline Top 1000 & 0.664 & 13.793 & 6.321 \\
\hline Top 2000 & 0.633 & 23.529 & 7.243 \\
\hline Top 3000 & 0.575 & 20.915 & 5.326 \\
\hline Top 4000 & 0.001 & 14.216 & 3.530 \\
\hline Top 5000 & $\mathbf{0 . 5 9 7}$ & $\mathbf{7 . 4 5 1}$ & $\mathbf{2 . 0 0 4}$ \\
\hline
\end{tabular}

Figure 12. Error of first-digit distributions of Video Uploads for top 5000 most-viewed channels 
Next, we evaluated the error of observed Total Views first-digit distribution for the top 1000 most-viewed YouTube channels. From the digits 1 to 9 , the error of each digit distribution was between $6.329 \%$ and $117.600 \%$, with the average error being $47.149 \%$. The error of the first-digit distribution of the top 2000 most-viewed YouTube channels had a minimum of $8.800 \%$ error and a maximum of $78.261 \%$ error, with the average error being $46.878 \%$. The error of the first-digit distribution of the top 3000 most-viewed YouTube channels had a minimum of $10.417 \%$ error and a maximum of $92.802 \%$ error, with the average error being $60.771 \%$. The error of the first-digit distribution of the top 4000 most-viewed YouTube channels had a minimum of $17.188 \%$ error and a maximum of $164.674 \%$ error, with the average error being $70.749 \%$. The error of the first-digit distribution of the top 5000 most-viewed YouTube channels had a minimum of $33.289 \%$ error and a maximum of $147.843 \%$ error, with the average error being $83.519 \%$. These results are also shown in Figure 13.

\begin{tabular}{|c|c|c|c|}
\hline $\begin{array}{c}\text { Most-Subscribed } \\
\text { Channels }\end{array}$ & Minimum Error (\%) & Maximum Error (\%) & Average Error (\%) \\
\hline Top 1000 & 6.329 & 117.600 & 47.149 \\
\hline Top 2000 & 8.800 & 78.261 & 46.878 \\
\hline Top 3000 & 10.417 & 92.802 & 60.771 \\
\hline Top 4000 & 17.188 & 164.674 & 70.749 \\
\hline Top 5000 & $\mathbf{3 3 . 2 8 9}$ & $\mathbf{1 4 7 . 8 4 3}$ & $\mathbf{8 3 . 5 1 9}$ \\
\hline
\end{tabular}

Figure 13. Error of first-digit distributions of Total Views for top 5000 most-viewed channels

Thirdly, we evaluated the error of observed Total Subscriptions first-digit distribution for the top 1000 most-viewed YouTube channels. From the digits 1 to 9 , the error of each digit distribution was between $2.070 \%$ and $47.436 \%$, with the average error being $18.217 \%$. The error of the first-digit distribution of the top 2000 most-viewed YouTube channels had a minimum of $6.844 \%$ error and a maximum of $25.816 \%$ error, with the average error being $16.178 \%$. The error of the first-digit distribution of the top 3000 most-viewed YouTube channels had a minimum of $0.739 \%$ error and a maximum of $24.811 \%$ error, with the average error being $13.012 \%$. The error of the first-digit distribution of the top 4000 most-viewed YouTube channels had a minimum of $0.047 \%$ error and a maximum of $23.815 \%$ error, with the average error being $11.474 \%$. The error of the first-digit distribution of the top 5000 most-viewed YouTube channels had a minimum of $0.497 \%$ error and a maximum of $23.815 \%$ error, with the average error being $10.604 \%$. These results are also shown in Figure 14.

\begin{tabular}{|c|c|c|c|}
\hline $\begin{array}{c}\text { Most-Subscribed } \\
\text { Channels }\end{array}$ & Minimum Error (\%) & Maximum Error (\%) & Average Error (\%) \\
\hline Top 1000 & 2.070 & 47.436 & 18.217 \\
\hline Top 2000 & 6.844 & 25.816 & 16.178 \\
\hline Top 3000 & 0.739 & 24.811 & 13.012 \\
\hline Top 4000 & 0.047 & 25.566 & 11.474 \\
\hline Top 5000 & $\mathbf{0 . 4 9 7}$ & $\mathbf{2 3 . 8 1 5}$ & $\mathbf{1 0 . 6 0 4}$ \\
\hline
\end{tabular}

Figure 14. Error of first-digit distributions of Total Subscriptions for top 5000 most-viewed channels 


\section{Analysis}

From the error analysis above, it can be seen that the error of first-digit distributions for Total Subscriptions when using Total Subscriptions as the control variable is much higher than the error of first-digit distributions for Total Views and Video Uploads, which leads us to believe that Total Subscriptions, when using the data from the top 5000 most-subscribed channels, can be seen as "artificially" generated and therefore doesn't fit Benford's Law, which would make sense because we are artificially selecting the top 5000 most-subscribed channels. However, the other two variables (the ones that aren't used as the control variable) have first-digit distributions that fit Benford's Law even though the two other variables are also extracted from the top 5000 artificially selected channels. This phenomenon is also seen for the analysis of the top 5000 most-viewed channels.

This phenomenon was further investigated through the use of smaller groups of channels within the top 5000 channels, including the top 1000, 2000, 3000, and 4000. From these tests, we noticed how there was a general trend of average errors decreasing as the number of channels increased for groups that fit Benford's Law, but groups that analyzed the same variable as the control variable still had larger errors while the groups that analyzed a variable different from the control variable generally had smaller errors. This leads us to believe that the two other variables (Total Views and Total Variables in the case of the top 5000 most-subscribed channels and Total Uploads and Total Subscriptions in the case of the top 5000 most-viewed channels) are not influenced by how the channels are selected, and are therefore not correlated with the control variable, allowing them to be "randomly" generated, which fits the requirements of Benford's Law.

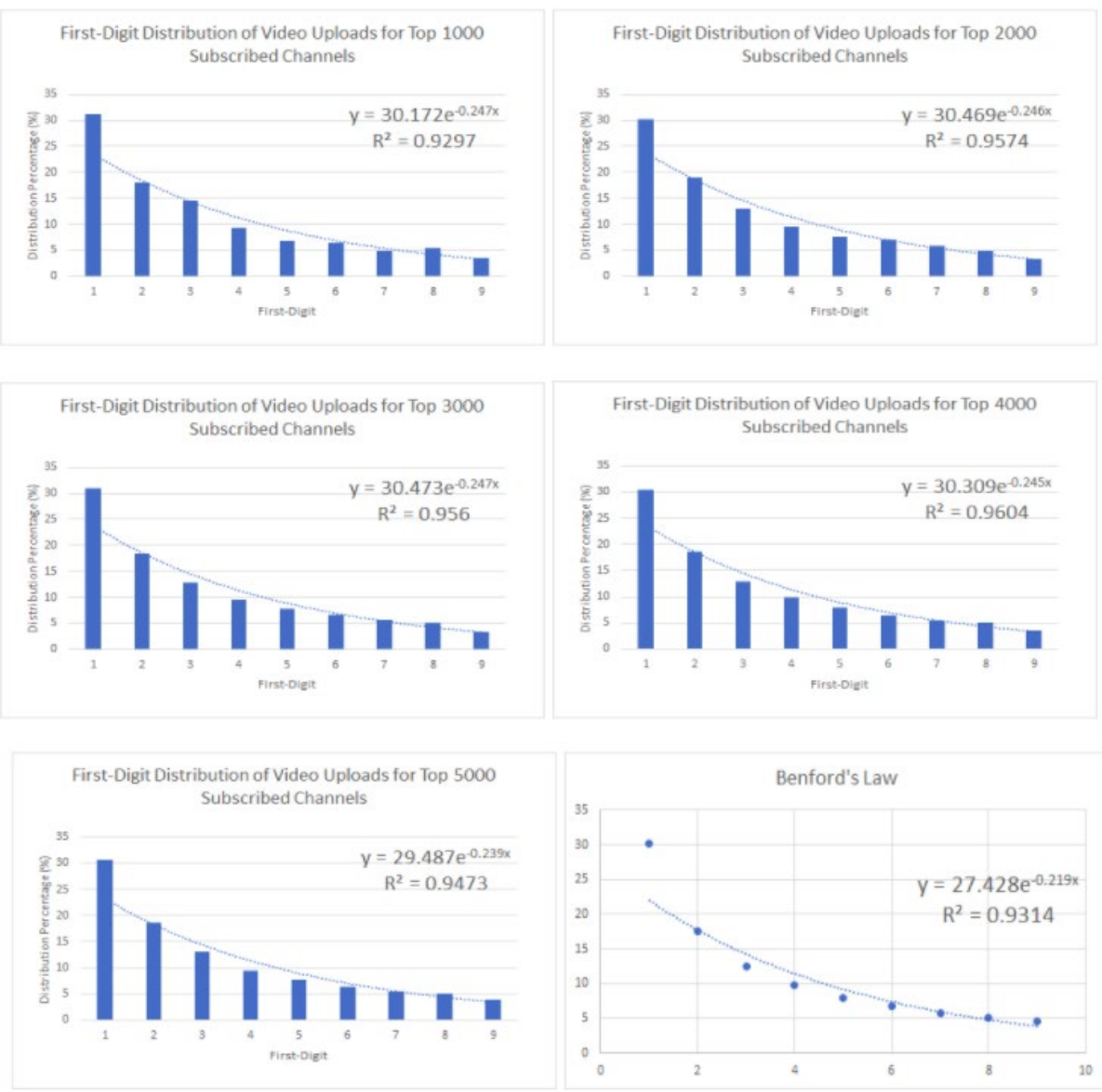

Figure 15. First-digit distribution of top 5000 video uploads (ranked) curve fitted with exponential models. Five graphs above are respectively the result of top 1000, 2000, 3000, 4000, 5000 subscribed channels and Benford's Law. 


\section{Verifying Benford's Law with exponential model}

\section{Curve fitting with exponential model}

Figures 15, 16, and 17 show the results of curve fitting the data, and it can be seen that Total Subscriptions of the top-5000 most subscribed channels had many different curving results that highly deviated from the expected results of Benford's Law while Video Uploads and Total Views generally have a and b values within a close range of the expected values.
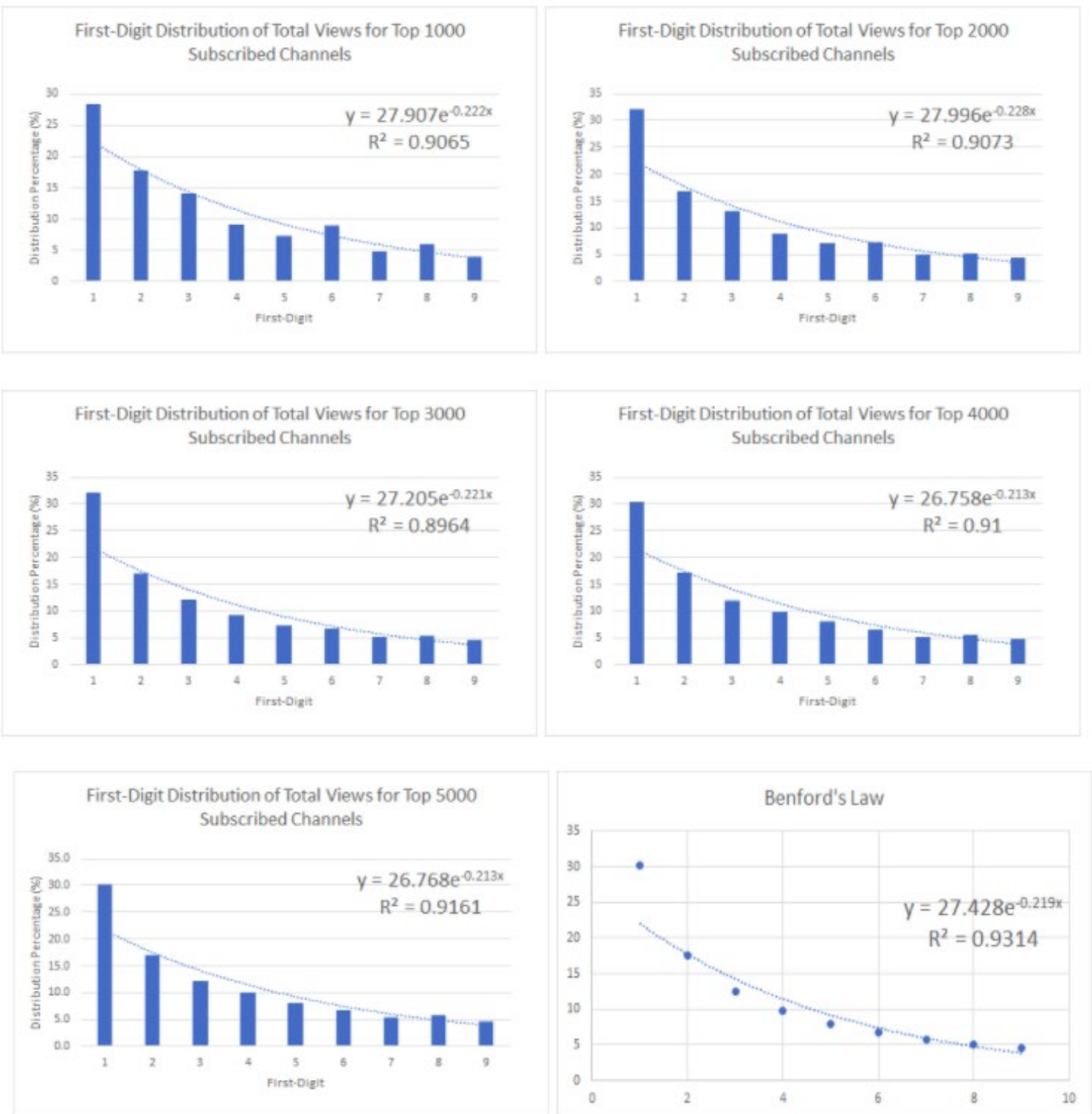

Figure 16. First-digit distribution of top 5000 total video views (ranked) curve fitted with exponential models. Five graphs above are respectively the result of top 1000, 2000, 3000, 4000, 5000 subscribed channels and Benford's Law. 
First-Digit Distribution of Total Subscriptions for Top 1000 Subscribed Channels

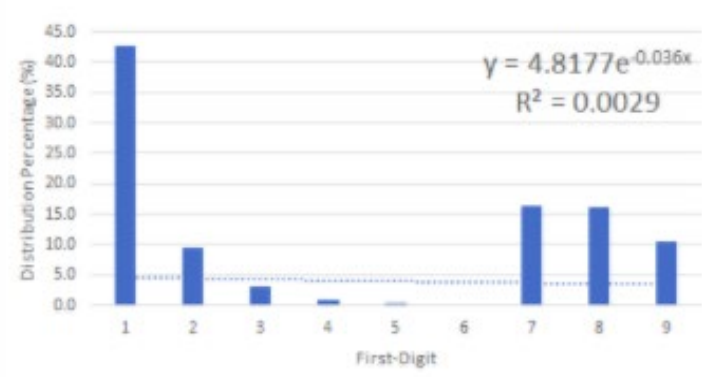

First-Digit Distribution of Total Subscriptions for Top 3000 Subscribed Channels

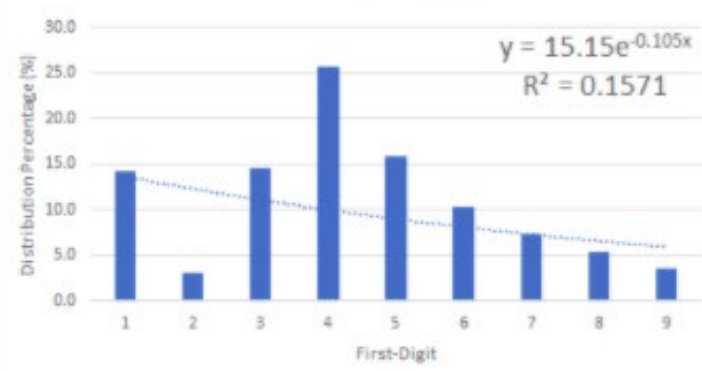

First-Digit Distribution of Total Subscriptions for Top 5000 Subscribed Channels

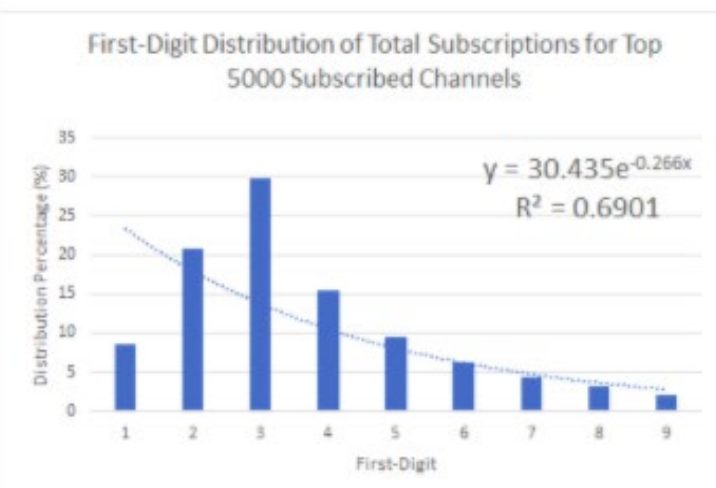

First-Digit Distribution of Total Subscriptions for Top 2000 Subscribed Channels

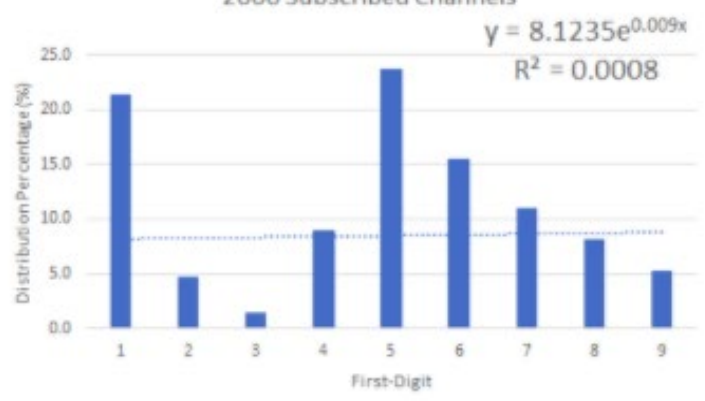

First-Digit Distribution of Total Subscriptions for Top 4000 Subscribed Channels

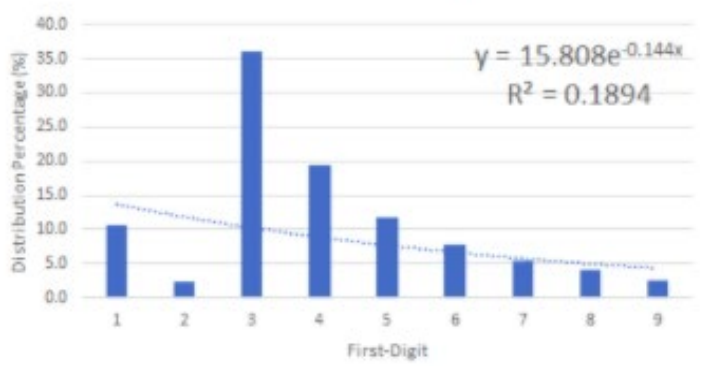

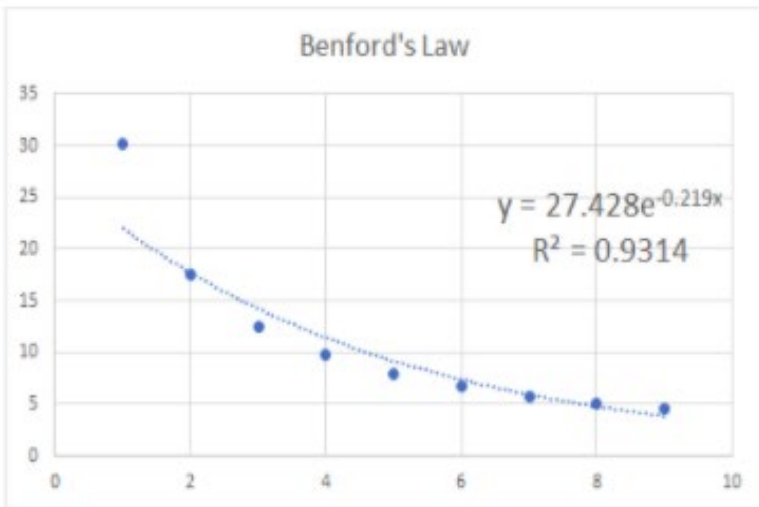

Figure 17. First-digit distribution of top 5000 subscribed channels (ranked) curve fitted with exponential models. Five graphs above are respectively the result of top 1000, 2000, 3000, 4000, 5000 subscribed channels and Benford's Law.

Figures 18, 19, and 20 show the results of curve fitting the data for the top 5000 most-viewed channels. It can be seen the Total Views has distributions that generally don't fit the expected curve of Benford's Law, while Total Subscriptions and Video Uploads slightly deviated from the expected curve but still distribute patterns fitting Benford's Law. 

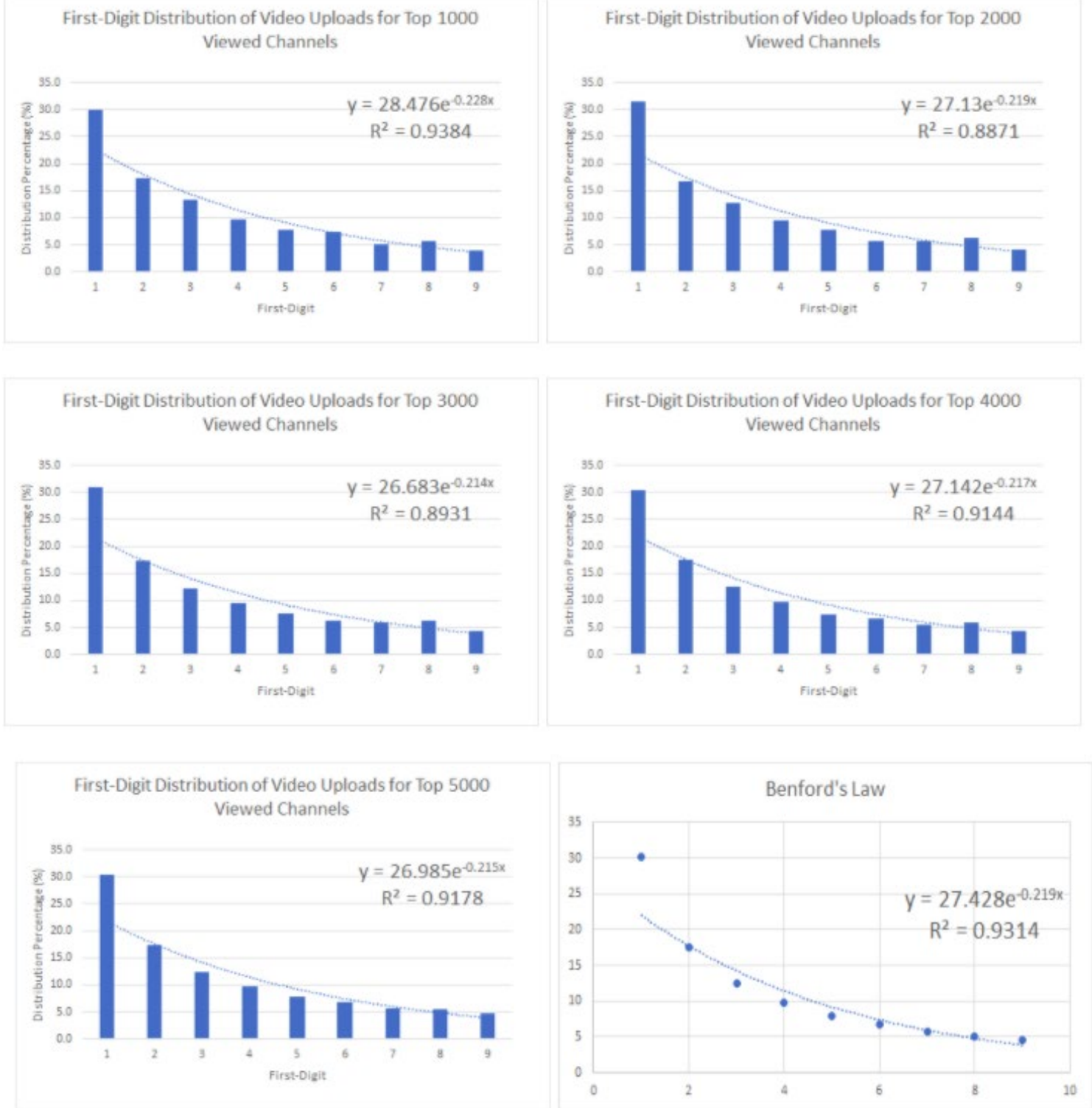

Figure 18. First-digit distribution of top 5000 video uploads (ranked) curve fitted with exponential models. Five graphs above are respectively the result of top 1000, 2000, 3000, 4000, 5000 total video views and Benford's Law. 

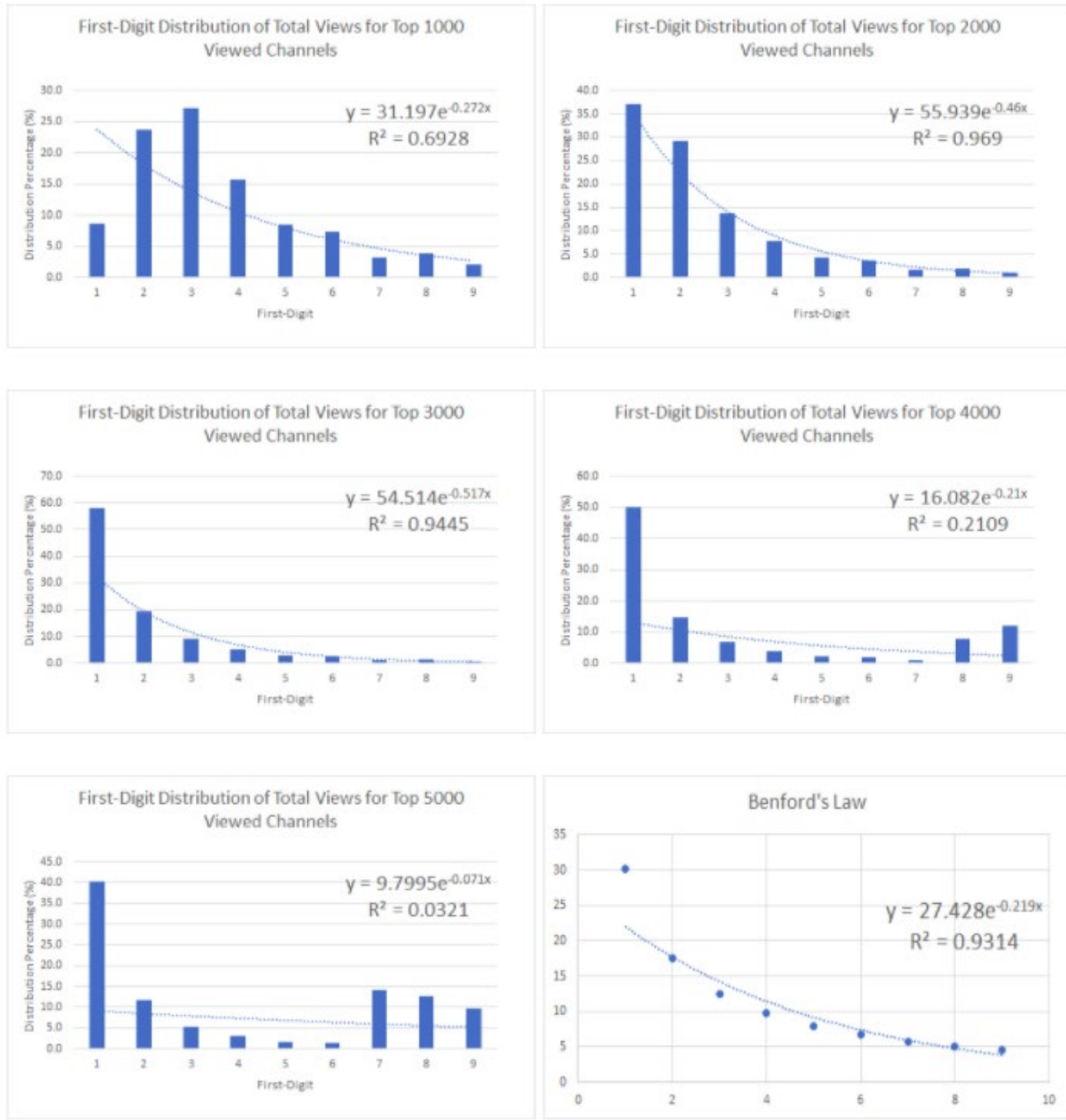

Figure 19. First-digit distribution of top 5000 total video views (ranked) curve fitted with exponential models. Five graphs above are respectively the result of top 1000, 2000, 3000, 4000, 5000 total video views and Benford's Law. 

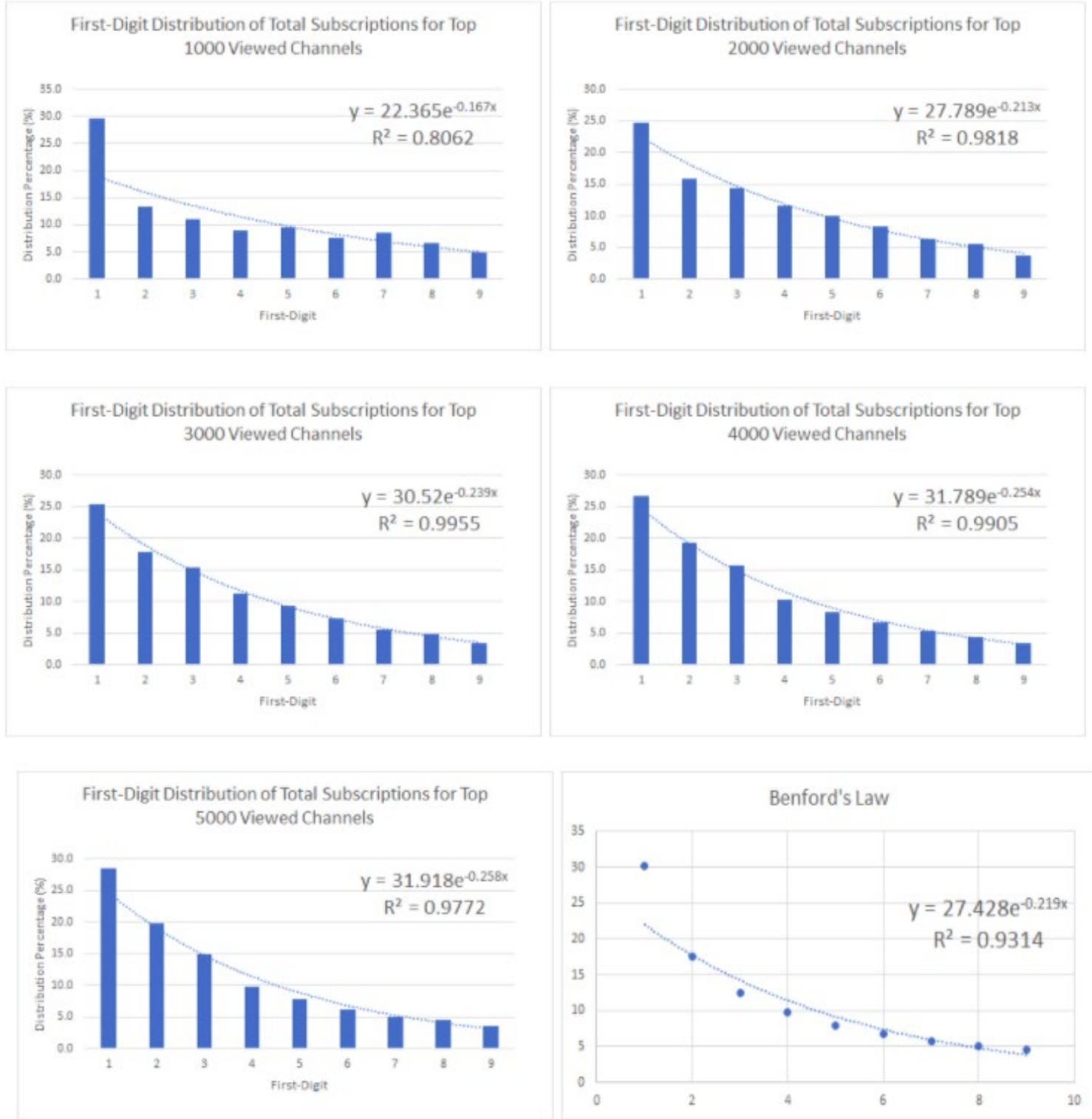

Figure 20. First-digit distribution of top 5000 subscribed channels (ranked) curve fitted with exponential models. Five graphs above are respectively the result of top 1000, 2000, 3000, 4000, 5000 total video views and Benford's Law.

\section{Error of $a$ and $b$ values in Comparison with Expected values}

From Figures 21 and 22, it can be seen that errors of a and b values resulting from curve fitting are smaller for Total Views and slightly larger for Video Uploads but all less than $20 \%$ percent, while the errors of a and $b$ values for Total Subscriptions as shown in Figure $\mathbf{2 3}$ easily exceeded 20\%. This corroborates our analysis of first-digit distributions above that groups analyzing the same variables as their control variables won't fit Benford's Law. 


\begin{tabular}{|c|c|c|}
\hline $\begin{array}{c}\text { Most-Subscribed } \\
\text { Channels }\end{array}$ & Error of a value (\%) & Error of b value (\%) \\
\hline Top 1000 & 10.004 & 12.785 \\
\hline Top 2000 & 11.087 & 12.329 \\
\hline Top 3000 & 11.102 & 12.785 \\
\hline Top 4000 & 10.504 & 11.872 \\
\hline Top 5000 & $\mathbf{7 . 5 0 7}$ & $\mathbf{9 . 1 3 2}$ \\
\hline
\end{tabular}

Figure 21. Error of a and $b$ values of groups taking Video Uploads for top 5000 most-subscribed channels in comparison with expected a and $\mathrm{b}$ for Benford's Law

\begin{tabular}{|c|c|c|}
\hline $\begin{array}{c}\text { Most-Subscribed } \\
\text { Channels }\end{array}$ & Error of a value (\%) & Error of b value (\%) \\
\hline Top 1000 & 1.746 & 1.370 \\
\hline Top 2000 & 2.071 & 4.110 \\
\hline Top 3000 & 0.813 & 0.913 \\
\hline Top 4000 & 2.443 & 2.740 \\
\hline Top 5000 & $\mathbf{2 . 4 0 6}$ & $\mathbf{2 . 7 4 0}$ \\
\hline
\end{tabular}

Figure 22. Error of a and b values of groups taking Total Views for top 5000 most-subscribed channels in comparison with expected $a$ and $b$ for Benford's Law

\begin{tabular}{|c|c|c|}
\hline $\begin{array}{c}\text { Most-Subscribed } \\
\text { Channels }\end{array}$ & Error of a value (\%) & Error of b value (\%) \\
\hline Top 1000 & 82.434 & 83.562 \\
\hline Top 2000 & 70.381 & 95.890 \\
\hline Top 3000 & 44.764 & 52.055 \\
\hline Top 4000 & 42.365 & 34.247 \\
\hline Top 5000 & $\mathbf{1 0 . 9 6 3}$ & $\mathbf{2 1 . 4 6 1}$ \\
\hline
\end{tabular}

Figure 23. Error of a and $b$ values of groups taking Total Subscriptions for top 5000 most-subscribed channels in comparison with expected a and $\mathrm{b}$ for Benford's Law 
From Figures 24 and 26, it can be seen that errors of a and $b$ values resulting from curve fitting are smaller for Video Uploads and slightly larger for Total Subscriptions but are mostly smaller than $20 \% \%$. This corroborates with our analysis above. For Figure 25, the Total Views for the top 5000 most-viewed channels, the errors exceeded 20\%\% easily, with some reaching above $100 \%$, showing that they don't fit Benford's Law.

\begin{tabular}{|c|c|c|}
\hline $\begin{array}{c}\text { Most-Subscribed } \\
\text { Channels }\end{array}$ & Error of a value (\%) & Error of b value (\%) \\
\hline Top 1000 & 3.821 & 4.110 \\
\hline Top 2000 & 1.086 & 0.000 \\
\hline Top 3000 & 2.716 & 2.283 \\
\hline Top 4000 & 1.043 & 0.913 \\
\hline Top 5000 & $\mathbf{1 . 6 1 5}$ & $\mathbf{1 . 8 2 6}$ \\
\hline
\end{tabular}

Figure 24. Error of a and b values of groups taking Video Uploads for top 5000 most-viewed channels in comparison with expected a and $b$ for Benford's Law

\begin{tabular}{|c|c|c|}
\hline $\begin{array}{c}\text { Most-Subscribed } \\
\text { Channels }\end{array}$ & Error of a value (\%) & Error of b value (\%) \\
\hline Top 1000 & 13.741 & 24.201 \\
\hline Top 2000 & 103.949 & 110.046 \\
\hline Top 3000 & 98.753 & 136.073 \\
\hline Top 4000 & 41.366 & 4.110 \\
\hline Top 5000 & $\mathbf{6 4 . 2 7 4}$ & $\mathbf{6 7 . 5 8 0}$ \\
\hline
\end{tabular}

Figure 25. Error of a and $b$ values of groups taking Total Views for top 5000 most-viewed channels in comparison with expected $\mathrm{a}$ and $\mathrm{b}$ for Benford's Law

\begin{tabular}{|c|c|c|}
\hline $\begin{array}{c}\text { Most-Subscribed } \\
\text { Channels }\end{array}$ & Error of a value (\%) & Error of b value (\%) \\
\hline Top 1000 & 18.459 & 23.744 \\
\hline Top 2000 & 1.316 & 2.740 \\
\hline Top 3000 & 11.273 & 9.132 \\
\hline Top 4000 & 15.900 & 15.982 \\
\hline Top 5000 & $\mathbf{1 6 . 3 7 0}$ & $\mathbf{1 7 . 8 0 8}$ \\
\hline
\end{tabular}

Figure 26. Error of a and b values of groups taking Total Subscriptions for top 5000 most-viewed channels in comparison with expected a and $\mathrm{b}$ for Benford's Law 


\section{Analysis}

The experimental results show that fitting first-digit distributions of a dataset with an exponential model can be used to evaluate how closely a dataset fits Benford's Law. Also, for most of the datasets that follow Benford's Law, as shown in Figures 21, 22, 24, and 26, the lowest error of a and b values typically occurs at groups with higher amounts of channels (4000 or 5000). This most likely indicates that datasets with larger quantities of data are more likely to follow the expected values of Benford's Law more closely. From Figure 23 and Figure 25, it can also be seen that even for datasets that don't fit Benford's Law according to its first-digit distributions, modeling these distributions with an exponential model will allow us to observe whether it has a similar trend to Benford's Law (a decreasing trend of frequency as the digit increases from 1 to 9 ).

\section{Conclusion and Implications}

In this paper, we have successfully utilized social media data to investigate Benford's Law. Using YouTube channel data taken from SocialBlade, we analyzed three variables- Total Subscriptions, Total Views, and Video Uploads- for each channel to verify if YouTube data fits Benford's Law and whether it is artificial or not. When taking Total Subscription data for the top 5000 most-subscribed channels, the first-digit distribution of Total Subscriptions doesn't fit Benford's Law, but the other two variables, Video Uploads and Total Views obtained fit. The same happens when taking Total Views for the top 5000 most-viewed channels. Thus, we can hypothesize that when analyzing variable A's first digit distribution for the top channels ranked with variable A, the first-digit distribution of variable A will not fit Benford's Law, while variables B and C's first-digit distribution obtained from variable A will fit and therefore are not artificial. In order to prove this hypothesis, we changed our number of channels to the top 1000, 2000, 3000, 4000, in addition to 5000 and found out that groups with different numbers of channels produce the same results. Otherwise, we also utilize an exponential model $y=a e^{-b x}$ to mathematically fit all the data. Results show that the a value of Benford's law is 27.428 and the b value is 0.219 . If the results of fitting first-digit distribution graphs produce $a$ and $b$ values that are closer to the expected $a$ and $b$ from Benford's Law, it is more likely that the data fits Benford's Law and isn't artificial. This method can be adopted to verify whether the first-digit distribution of data fits Benford's Law. In the future, we will use this proposed model to verify whether or not other datasets fit Benford's Law, and whether they are artificial or not.

\section{Acknowledgments}

We would like to thank our advisor Hsin-Ye (David) Chen for helping us with this project.

\section{References}

[1] Berger, Arno, and Theodore P. Hill. “A Basic Theory of Benford's Law.” Probability Surveys, vol. 8, no. none, 2011, doi:10.1214/11-ps175.

[2] Kruger, Paul, and Sarma Yadavalli. “THE POWER OF ONE: BENFORD'S LAW.” South African Journal of Industrial Engineering, vol. 28, no. 2, 2017, doi:10.7166/28-2-1753.

[3] Jamain, Adrien. "Benford's Law.” Sept. 2001.

[4] Berger, Arno, and Theodore P. Hill. An Introduction to Benford's Law. Princeton U.P., 2015.

[5] Romero-Roch'in, V.. “A derivation of Benford's Law ... and a vindication of Newcomb.” (2009). 